\title{
Socio-economic differences in cardiometabolic risk markers are mediated by diet and body fatness in 8- to 11 -year-old Danish children: a cross-sectional study
}

\author{
Hanne Hauger ${ }^{1, *}$, Margit V Groth ${ }^{2}$, Christian Ritz ${ }^{1}$, Anja Biltoft-Jensen ${ }^{2}$, Rikke Andersen ${ }^{2}$, \\ Stine-Mathilde Dalskov ${ }^{1}$, Mads F Hjorth ${ }^{1}$, Anders Sjödin ${ }^{1}$, Arne Astrup ${ }^{1}$, Kim F Michaelsen ${ }^{1}$ \\ and Camilla T Damsgaard ${ }^{1}$ \\ 'Department of Nutrition, Exercise and Sports, Faculty of Science, University of Copenhagen, Rolighedsvej 26, 1958 \\ Frederiksberg C, Denmark: ${ }^{2}$ Division of Nutrition, National Food Institute, Technical University of Denmark, Søborg, \\ Denmark
}

Submitted 14 June 2015: Final revision received 19 November 2015: Accepted 16 December 2015: First published online 1 March 2016

\begin{abstract}
Objective: To explore whether socio-economic differences exist in cardiometabolic risk markers in children and whether lifestyle-related factors potentially mediate these differences.

Design: Cross-sectional study including measurements of fasting blood lipids, glucose, homeostasis model assessment of insulin resistance (HOMA-IR), blood pressure and heart rate. Potential mediators examined were fat mass index (FMI); intakes of fruit, vegetables, dietary fibre and added sugar; whole-blood $n$ - 3 longchain PUFA (LCPUFA) as a biomarker of fish intake; and physical activity and sedentary time.

Setting: Nine primary schools in Denmark.

Subjects: Children aged 8-11 years ( $n$ 715).

Results: Children of parents with the shortest compared with longest education had higher TAG by $0 \cdot 12(95 \%$ CI $0 \cdot 04,0 \cdot 21) \mathrm{mmol} / \mathrm{l}$ and HOMA-IR by $0 \cdot 36(0 \cdot 10$, $0 \cdot 62)$, whereas children of parents with a vocational education had higher total cholesterol by $0 \cdot 14(0 \cdot 02,0 \cdot 27) \mathrm{mmol} / 1$ and LDL cholesterol by $0 \cdot 14(0 \cdot 03,0 \cdot 25)$ $\mathrm{mmol} / \mathrm{l}$ compared with children of parents with the longest education; all $P<0.05$. FMI explained $25 \%$ of the difference in TAG, $64 \%$ of the difference in HOMA-IR and $21-29 \%$ of the differences in cholesterols. FMI and whole-blood $n-3$ LCPUFA combined explained $42 \%$ of the difference in TAG, whereas FMI, whole-blood n-3 LCPUFA and dietary fibre explained $89 \%$ of the difference in HOMA-IR. Conclusions: Socio-economic differences were present in blood lipids and insulin resistance among 8- to 11-year-olds and were mediated by body fatness, wholeblood n-3 LCPUFA and dietary fibre. These lifestyle factors may be targets in public initiatives to reduce socio-economic differences. Confirmation in longitudinal studies and trials is warranted.
\end{abstract}

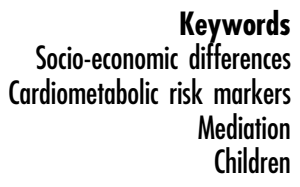

Socio-economic inequalities in CVD mortality contribute substantially to the inequality in all-cause mortality in most European countries ${ }^{(1)}$. Evidence suggests that CVD originates in childhood $^{(2,3)}$ and that a low socio-economic position early in life increases later CVD risk independent of one's adult socio-economic position ${ }^{(4)}$. However, we lack knowledge on modifiable factors that may help reduce socio-economic differences in cardiometabolic risk from childhood. It is, therefore, relevant to investigate whether socio-economic differences in cardiometabolic risk markers are present in childhood and whether lifestyle factors play a mediating role in these differences.
Some of the relatively few studies that have examined cardiometabolic inequalities in childhood have shown higher blood pressure ${ }^{(5)}$, insulin resistance ${ }^{(6,7)}$ and a more adverse lipid profile ${ }^{(8)}$ among the lowest socio-economic groups. Obesity is a strong driver of cardiometabolic derangements, also in childhood ${ }^{(9)}$, and many studies of children from high-income countries have shown that low socio-economic position is associated with overweight or obesity ${ }^{(10)}$. Observational studies in children and adolescents have shown that inequalities in blood pressure and insulin resistance were mediated by fat mass and BMI, respectively ${ }^{(5,11)}$. BMI is commonly used as a 
proxy measure of body fatness, but the fat mass index $(\text { FMI })^{(12)}$ may be better suited for investigating such associations, as it is not influenced by the amount of lean mass. Diets low in added sugar and rich in fruit, vegetables, dietary fibre and $n-3$ long-chain PUFA (LCPUFA) from fish have been linked to better cardiometabolic profiles in children ${ }^{(13,14)}$ and lower CVD risk in adults ${ }^{(15-18)}$. Children of lower socio-economic backgrounds have been shown to eat less healthy diets ${ }^{(19,20)}$, which could contribute to inequalities in cardiometabolic risk markers between children of higher and lower socioeconomic positions. Physical activity and sedentary behaviour may be other potential contributors as higher moderate-to-vigorous physical activity (MVPA) has been associated with better cardiometabolic profiles in schoolchildren $^{(21)}$, whereas sedentary behaviour has been linked to increased CVD risk in adults ${ }^{(22)}$. Dietary components, physical activity and sedentary time may affect cardiometabolic risk markers directly or via body fatness. However, to our knowledge, no studies have examined whether socio-economic differences in cardiometabolic risk profiles in children from high-income countries are mediated by dietary components and objectively measured physical activity or sedentary time. Also, it has not been investigated whether dietary components and physical activity play a mediating role independent of body fatness.

The aims of the present study were (i) to explore whether socio-economic differences were present in various cardiometabolic risk markers among 8- to 11-year-old Danish children; and (ii) if differences were observed, to explore whether the following were potential independent mediators: FMI; dietary components such as fruit, vegetables, fibre and added sugar; whole-blood n-3 LCPUFA (as a biomarker of fish intake); and MVPA and sedentary time.

\section{Methods}

\section{Study design and population}

The present cross-sectional study used baseline data collected during 2011 within the OPUS (Optimal wellbeing, development and health for Danish children through a healthy New Nordic Diet) School Meal Study. The primary aims of the OPUS study were to examine the effect of Nordic school meals on metabolic health and concentration performance in third- and fourth-grade pupils. A detailed description of the study design and methods has been published previously ${ }^{(23)}$. In total, 1021 pupils from forty-six school classes at nine schools were invited, and among these 834 (82\%) children participated in the OPUS School Meal Study. The present study included the 715 children from whom baseline data on household education, at least one cardiometabolic risk marker and all potential mediators and relevant covariates were available. The cardiometabolic risk markers included were blood cholesterols, TAG, glucose, homeostasis model assessment of insulin resistance (HOMA-IR), blood pressure and heart rate. Potential mediators examined were FMI; intakes of fruit, vegetables, dietary fibre and added sugar; whole-blood $n$-3 LCPUFA as a biomarker of fish intake; and MVPA and sedentary time. Additionally, waist circumference and BMI-for-age $Z$-scores were included as mediators to substantiate the results.

\section{Interviews on socio-economic position, ethnicity and puberty}

At baseline, each family was interviewed about socioeconomic factors and demographics. We used the highest level of education obtained in the household as the indicator of socio-economic position, since education most consistently predicts CVD risk factors in children and adults compared with income and occupation, both in Denmark and internationally ${ }^{(8,24-26)}$. The level of education was divided into categories ${ }^{(27)}$ based on the International Standard Classification of Education (ISCED): lower secondary ( $\leq 10$ years), upper secondary (12 years), vocational (12 years), short higher (14 years), medium higher (15-16 years) and long higher education $(\geq 17$ years). Lower secondary education included compulsory schooling; upper secondary education included programmes qualifying for higher educations, e.g. high school; and the vocational category included practical educations, e.g. sales assistant, carpenter, etc. The short higher education category included e.g. market economist and laboratory technician; medium higher education corresponded to bachelor degree; and the long higher education category included mainly university master and $\mathrm{PhD}$ degrees. We pooled data from children in the upper secondary ( $n$ 20) and vocational education categories ( $n$ 231) since they did not differ on any cardiometabolic risk markers; the group is referred to as 'vocational education'. Children were defined as immigrants/ descendants if all grandparents and at least one parent were born outside Denmark. Pubertal status was selfevaluated by the child using Tanner stages, which are based on breast development in girls and pubic hair in boys ${ }^{(28)}$. Since only forty-six children had reached Tanner stages 3-5 we used only two pubertal categories: 'not entered puberty' (Tanner stage 1) and 'entered puberty' (Tanner stages 2-5).

\section{Recording of diet and physical activity}

With parental assistance, children recorded their dietary intake for seven consecutive days using web-based dietary assessment software developed for the age group ${ }^{(29)}$. Dietary variables included in the present study were intake of fruit (excluding fruit juice, but including nuts), vegetables (excluding potatoes), dietary fibre and fish measured in $\mathrm{g} / 10 \mathrm{MJ}$ as well as intake of added sugar measured in percentage of total daily energy intake (E\%). 
Only children with at least four days of dietary recording were included in the analyses. The ratio between energy intake (EI) and estimated $\mathrm{BMR}^{(30)}$ was used to categorize children as under-reporters (EI:BMR $\leq 1.05$ ), acceptable reporters (EI:BMR $>1.05$ and $<2.29$ ) and over-reporters (EI:BMR $\geq 2 \cdot 29$ ) of energy intake ${ }^{(31)}$. Physical activity was measured using an accelerometer (GT3X or GT3X+; ActiGraph, Pensacola, FL, USA) worn tightly at the right hip and affixed with an elastic belt, as previously described $^{(32)}$. Recordings of $\geq 10 \mathrm{~h}$ monitor wear time for at least three weekdays and at least one weekend day were included in the analyses. We used 0-100 counts/min and $\geq 2296$ counts/min as the cut-offs for time spent sedentary and in MVPA, respectively ${ }^{(33)}$.

\section{Clinical examinations}

Clinical examinations were conducted in a double-decker truck equipped as a mobile laboratory and parked at the schools. Except for body composition, all measurements were performed in the morning after an overnight fast. A total of twenty-one children did not fast; eight of these had consumed only chewing gum or single bites of food. During the anthropometric measurements, the children were wearing light clothing and had emptied their bladders. Height was measured to the nearest $0 \cdot 1 \mathrm{~cm}$ (CMS Weighing Equipment, London, UK) while the examiner made sure that the children's heads were in the Frankfurt horizontal plane and calculated as the mean of three repeated measurements. Body weight was measured to the nearest $0 \cdot 1 \mathrm{~kg}$ on a digital scale (Tanita $800 \mathrm{~S}$, Arlington Heights, IL, USA). BMI was calculated as: [body weight (kg)]/[height $(\mathrm{m})]^{2}$, and sex- and age-adjusted $Z$-scores for BMI were calculated using the WHO Growth Reference 2007 for children aged 5-19 years ${ }^{(34)}$. The prevalence of underweight, overweight and obesity was based on ageand sex-specific cut-offs defined by centiles passing through BMI of $18.5,25.0$ and $30.0 \mathrm{~kg} / \mathrm{m}^{2}$, respectively, at 18 years $^{(35,36)}$. Waist circumference was measured at the level of the umbilicus and the mean of three repeated measurements was used. Total fat mass was assessed by whole-body dual-energy X-ray absorptiometry scan (Lunar Prodigy Pro ${ }^{\mathrm{TM}}$; GE Medical Systems, Madison, WI, USA) completed on the same day as the other measurements. Most children had a standardized breakfast prior to the scanning. FMI was calculated as: [fat mass (kg)]/[height $(\mathrm{m})]^{2}$. Blood pressure and heart rate were measured three times in the supine position after $10 \mathrm{~min}$ of rest using an automated device (ProBP 3400 Sure BP; Welch Allyn Inc., Skaneateles Falls, NY, USA). A second device was used for children with arm circumferences $>22 \mathrm{~cm}$ (UA-787 Plus; A\&D Medical, Tokyo, Japan). The mean of the last two measurements was used.

A $35 \mathrm{ml}$ fasting venous blood sample was drawn from the forearm. Whole-blood glucose concentrations were analysed immediately after sampling, and remaining blood was stored at $-80^{\circ} \mathrm{C}$ for later analysis. Plasma total cholesterol, HDL cholesterol and TAG, as well as serum insulin, were analysed, and LDL cholesterol concentrations were calculated using Friedewald's equation, as previously described ${ }^{(37)}$. HOMA-IR was calculated as: [glucose $(\mathrm{mmol} / \mathrm{l}) \times$ insulin $(\mathrm{mIU} / \mathrm{l})] / 22 \cdot 5$. Whole-blood $n$-3 LCPUFA was measured as EPA + DHA by highthroughput GC and is presented as relative weight of the total fatty acids $(\mathrm{w} / \mathrm{w} \%)^{(38)}$.

\section{Statistical analyses}

Differences between the long higher education group (as reference) and the other education groups were evaluated by linear or logistic mixed-effects models including school and class as random effects to account for the clustering of pupils. Our exploratory analysis consisted of four steps inspired by the causal steps approach ${ }^{(39)}$. First, we assessed whether educational differences were present in the various cardiometabolic risk markers. Only cardiometabolic risk markers showing educational differences were included in the subsequent steps of the analysis. Second, we assessed whether educational differences were present in the potential mediators. Only potential mediators that showed educational differences were included in the subsequent step. Third, we tested potential associations between the cardiometabolic markers identified in the first step and potential mediators identified in the second step. Potential mediators that were associated with the cardiometabolic markers were included in the fourth step: for each cardiometabolic risk marker, we expanded the models from step 1 by including the relevant mediators identified in step 3. Mediators were included in the models individually and together to evaluate whether they were independent and hence to account for multicollinearity in the interpretations. For example, if a dietary component was still significant in the model after adjusting for body fatness, it was considered to be a mediator independent of body fatness. For each cardiometabolic risk marker, the absolute mediated effects were calculated as $\beta_{\text {from model }}$ without mediator(s) $-\beta_{\text {from model including mediator(s) }}$ and the proportions mediated as $\left[\left(\beta_{\text {from model without mediator(s) }}-\beta_{\text {from }}\right.\right.$ model including mediator(s) $\left.) / \beta_{\text {from model without mediator(s) }}\right] \times 100 \%$. The absolute mediated effects were evaluated using Sobel's test.

All models were adjusted for age and sex. Analyses of cardiometabolic risk markers were furthermore adjusted for height, puberty and a sex $\times$ puberty interaction. Additionally, analyses of HOMA-IR, glucose and blood lipids were adjusted for fasting status, and analyses of blood pressure and heart rate were adjusted for blood pressure device and ambient temperature. Analyses of FMI were additionally adjusted for puberty; analyses of self-reported dietary variables were adjusted for under-, acceptable and over-reporting of energy intake; and analyses of MVPA and sedentary time were additionally adjusted for a sex $\times$ age interaction. Associations between whole-blood $n$-3 LCPUFA and fish intake were evaluated 
using Pearson's correlation coefficient. Model assumptions were checked by visual inspection of residual and normal probability plots. When necessary, variables were transformed and results subsequently back transformed ${ }^{(40)}$. Statistical analyses were performed using the statistical software package Stata ${ }^{\circledR}$ version $12 \cdot 1$ and $\mathrm{R}$ version $3 \cdot 0 \cdot 2^{(41)}$. Statistical significance was established at $P<0 \cdot 05$, using two-sided tests.

\section{Results}

\section{Characteristics of the study population}

The age, height and sex distribution of the children did not differ between the long higher and the other education groups (Table 1). Overweight and obesity proportions were higher in all groups where parents had less than a long higher education level, and it was highest in children from the group whose parents had a lower secondary education level. Children in this group were also more likely to be immigrants or descendants and to have entered puberty, compared with the long higher education group. However, the pubertal difference was no longer significant after adjustment for BMI-for-age $Z$-score and sex. Under- and over-reporters of energy intake comprised $8.1 \%(n 58)$ and $1.7 \%(n$ 12) of the study population, respectively, but the proportions of under-reporters differed only between the lower secondary and long higher education groups $(31.4 \%$ v. $6.0 \%, P<0.001)$. Among the 834 children originally enrolled in the OPUS School Meal Study, the 715 children included in the present study did not differ from the 119 not included with regard to BMI, age or gender, but they were less likely to have parents with a lower secondary education $(P<0 \cdot 01$; data not shown).

\section{Educational differences in cardiometabolic risk markers}

The lower secondary education group had higher plasma TAG and HOMA-IR compared with the long higher education group, and the vocational education group had higher total and LDL cholesterol (Table 2). The short higher education group differed from the long higher education group only with respect to plasma TAG. Blood pressure, heart rate, plasma HDL cholesterol and glucose did not differ between the long higher and other education groups.

\section{Educational differences in potential mediators}

Children of parents with shorter educations had higher FMI, waist circumference, BMI-for-age $Z$-scores, lower intakes of vegetables and dietary fibre, lower whole-blood n-3 LCPUFA and higher intakes of added sugar compared with the long higher education group (Table 3). In contrast, these groups spent less time sedentary than the long higher education group. Generally, the educational

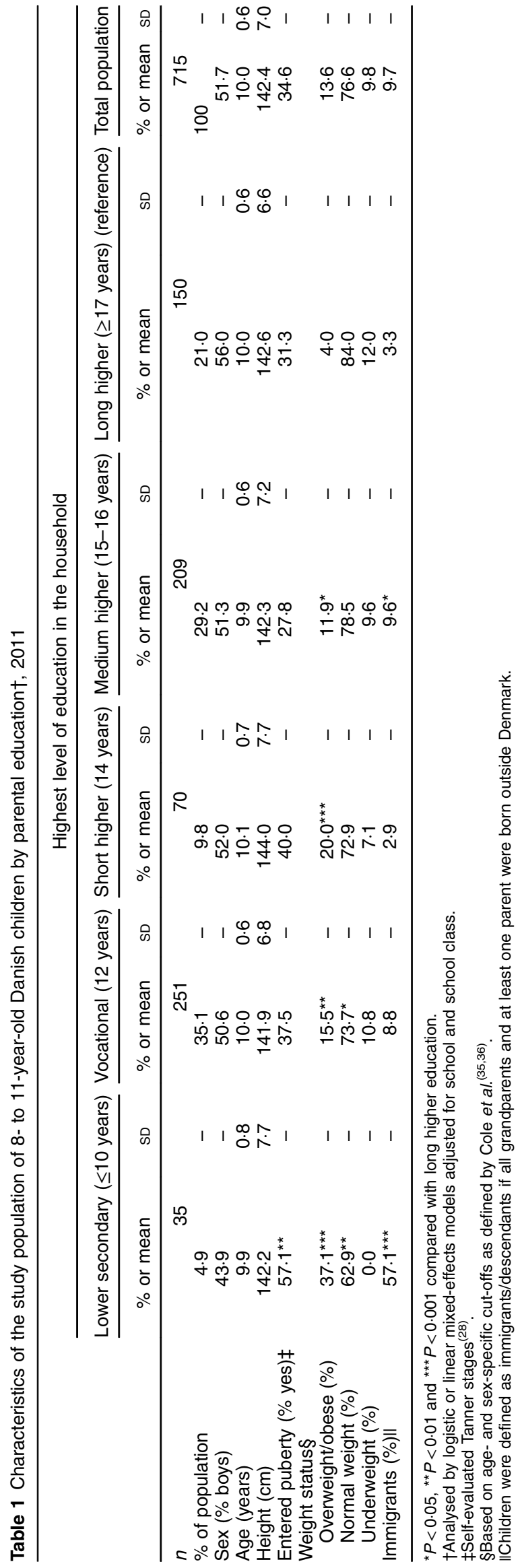


differences were largest between the lower secondary and the long higher education groups. Fruit intake and time spent in MVPA did not differ between the long higher and other education groups, nor did mean accelerometer wear time ( $P>0 \cdot 2$; data not shown).

Additional analyses showed that $76 \%$ of the children consumed fish and 15\% consumed fish oil during the dietary recording week. The percentage of children eating fish was lower among the children of parents with shorter educations (ranging from $67 \%$ in the lower secondary to $76 \%$ in the medium higher education group) compared with $87 \%$ in the long higher education group $(P<0.05)$. Mean frequency of fish-oil consumption was lower in the vocational, short higher and medium higher education groups $(0.3 \mathrm{~d} /$ week $)$ compared with $1 \mathrm{~d}$ /week in the long higher education group $(P<0 \cdot 001)$. The $n$-3 LCPUFA content in whole blood correlated positively with the reported intake of fish $(r=0.41, P<0.0001)$ and fatty fish $(r=0.39, P<0 \cdot 0001)$.

\section{Associations between potential mediators and cardiometabolic risk markers}

Among the putative mediators, FMI was positively associated with total and LDL cholesterol $(P<0 \cdot 01)$ and with plasma TAG and HOMA-IR $(P<0 \cdot 001)$. Whole-blood $n-3$ LCPUFA was negatively associated with plasma TAG $(P=0.002)$ and HOMA-IR $(P=0 \cdot 001)$. Dietary fibre intake was also negatively associated with HOMA-IR $(P=0 \cdot 001)$. Intakes of added sugar and vegetables and sedentary time were not associated with any of the cardiometabolic risk markers that showed educational differences. Accordingly, FMI, whole-blood $n$-3 LCPUFA and dietary fibre intake were evaluated further as potential mediators.

\section{Estimated mediated effects}

Table 4 shows how much of the educational differences in the cardiometabolic markers were accounted for by the mediators in absolute values and percentages. FMI accounted for part of the differences in the cholesterol concentrations, plasma TAG and HOMA-IR between the long higher education group and other groups with shorter educations. Whole-blood n-3 LCPUFA also accounted for part of the differences in plasma TAG and HOMA-IR, whereas dietary fibre intake accounted for part of the difference in HOMA-IR only.

The estimated mediated effects of the educational differences in plasma TAG and HOMA-IR became larger when the relevant mediators were entered together in the models. For example, FMI and whole-blood $n$-3 LCPUFA combined accounted for $0.05 \mathrm{mmol} / 1$ (42\%) out of the $0.12 \mathrm{mmol} / \mathrm{l}$ difference in TAG, whereas FMI, dietary fibre intake and whole-blood $n$-3 LCPUFA accounted for $0 \cdot 32$ points ( $89 \%$ ) out of the 0.36 point difference in HOMA-IR between the long higher and lower secondary education groups. All mediators remained associated with the cardiometabolic risk markers when included in the models 
Table 3 Differences in body fatness, dietary intakes, whole-blood $n-3$ LCPUFA, physical activity and sedentary time in 8- to 11 -year-old Danish children by parental education†, 2011

\begin{tabular}{|c|c|c|c|c|c|c|c|c|c|c|}
\hline & \multicolumn{10}{|c|}{ Highest education in the household } \\
\hline & \multicolumn{2}{|c|}{ Lower secondary ( $\leq 10$ years) } & \multicolumn{2}{|c|}{ Vocational (12 years) } & \multicolumn{2}{|c|}{ Short higher (14 years) } & \multicolumn{2}{|c|}{ Medium higher (15-16 years) } & \multicolumn{2}{|c|}{ Long higher ( $\geq 17$ years) (reference) } \\
\hline & $\beta$ & $95 \% \mathrm{Cl}$ & $\beta$ & $95 \% \mathrm{Cl}$ & $\beta$ & $95 \% \mathrm{Cl}$ & $\beta$ & $95 \% \mathrm{Cl}$ & Mean & $95 \% \mathrm{Cl}$ \\
\hline$n$ & \multicolumn{2}{|c|}{35} & \multicolumn{2}{|r|}{251} & \multicolumn{2}{|r|}{70} & \multicolumn{2}{|c|}{209} & \multicolumn{2}{|c|}{150} \\
\hline Body fatness $\ddagger$ & & & & & & & & & & \\
\hline FMl $\left(\mathrm{kg} / \mathrm{m}^{2}\right)$ & $1.34^{\star \star \star}$ & $0.76,1.98$ & $0.75^{\star \star \star}$ & $0.41,1.08$ & $0.78^{\star \star}$ & $0.31,1.24$ & $0.40^{*}$ & $0.06,0.75$ & $3 \cdot 16$ & $2.94,3.39$ \\
\hline WC (cm) & $5 \cdot 0^{\star \star \star}$ & $2 \cdot 8,7 \cdot 2$ & $2 \cdot 7^{\star \star \star}$ & $1.5,3.8$ & $3 \cdot 3^{\star \star \star}$ & $1 \cdot 6,4.9$ & $1.4^{\star}$ & $0.1,2 \cdot 6$ & $62 \cdot 1$ & $61 \cdot 1,63 \cdot 1$ \\
\hline BMl-for-age Z-score & $0.89^{\star \star \star}$ & $0.51,1.27$ & $0.38^{\star \star \star}$ & $0.17,0.59$ & $0.52^{\star \star \star}$ & $0.23,0.81$ & $0.28^{*}$ & $0.06,0.49$ & -0.17 & $-0.33,-0.00$ \\
\hline \multicolumn{11}{|l|}{ Dietary intakes§ } \\
\hline Fruit (g/10 MJ) & -19.4 & $-60 \cdot 1,21 \cdot 3$ & -18.0 & $-40 \cdot 0,4 \cdot 0$ & -9.0 & $-39.9,21.9$ & $-2 \cdot 7$ & $-25 \cdot 5,20 \cdot 1$ & 174.9 & $157 \cdot 2,192 \cdot 7$ \\
\hline Vegetables (g/10 MJ) & $-34.5^{\star}$ & $-66 \cdot 6,-2 \cdot 4$ & $-13 \cdot 4$ & $-30 \cdot 8,-4 \cdot 0$ & $-15 \cdot 8$ & $-39.9,8.3$ & $-18 \cdot 1^{*}$ & $-36 \cdot 0,-0 \cdot 1$ & $180 \cdot 4$ & $163 \cdot 6,197 \cdot 3$ \\
\hline Fibre $(g / 10 \mathrm{MJ})$ & $-3.5^{\star \star}$ & $-5 \cdot 6,-1 \cdot 5$ & $-1 \cdot 5^{\star}$ & $-2 \cdot 6,-0.4$ & $-1 \cdot 2$ & $-2 \cdot 7,0 \cdot 4$ & -0.9 & $-2 \cdot 1,0.2$ & $24 \cdot 2$ & $23 \cdot 1,25 \cdot 3$ \\
\hline Added sugar (E\%) & $2 \cdot 8^{\star \star \star}$ & $1.3,4.3$ & $1.9^{\star \star \star}$ & $1 \cdot 1,2 \cdot 8$ & $1 \cdot 2^{*}$ & $0.1,2 \cdot 4$ & $1.3^{\star *}$ & $0.5,2 \cdot 2$ & $9 \cdot 6$ & $8.9,10.3$ \\
\hline \multicolumn{11}{|l|}{ Biomarker of fish intake } \\
\hline$n$-3 LCPUFA (w/w\%) & $-0.78^{\star * *}$ & $-1 \cdot 12,-0.44$ & $-0.47^{\star * *}$ & $-0.66,-0.29$ & $-0 \cdot 36^{\star \star}$ & $-0.62,-0.11$ & $-0 \cdot 26^{* *}$ & $-0.45,-0.07$ & $3 \cdot 86$ & $3.65,4.08$ \\
\hline \multicolumn{11}{|l|}{ Physical activity\|l } \\
\hline Sedentary time $(\mathrm{min} / \mathrm{d})$ & $-31 \cdot 5^{\star *}$ & $-53 \cdot 4,-9 \cdot 6$ & $-17 \cdot 5^{\star \star}$ & $-29 \cdot 5,-5 \cdot 5$ & $-9 \cdot 6$ & $-26 \cdot 2,6 \cdot 9$ & -4.5 & $-16 \cdot 8,7 \cdot 9$ & $483 \cdot 5$ & $472 \cdot 7,494 \cdot 2$ \\
\hline MVPA (min/d) & -0.3 & $-7.5,6.8$ & $-3 \cdot 1$ & $-7.0,0.9$ & $-1 \cdot 1$ & $-6.5,4.4$ & -1.9 & $-6 \cdot 0,2 \cdot 2$ & 49.8 & $45 \cdot 4,54 \cdot 3$ \\
\hline
\end{tabular}

$n$-3 LCPUFA, $n$-3 long-chain PUFA (w/w\% of the total fatty acids measured in whole blood); FMI, fat mass index; WC, waist circumference; E\%, percentage of energy intake; MVPA, moderate-to-vigorous physical activity. ${ }^{*} P<0.05,{ }^{* *} P<0.01$ and ${ }^{* \star *} P<0.001$ compared with long higher education.

results are presented on the original scale.

łAnalyses of FMI, WC and BMI were additionally adjusted for puberty, and analyses of WC for height.

§Additionally adjusted for under-, acceptable and over-reporting of energy intake.

IAdditionally adjusted for an age $\times$ sex interaction. 
presented in Table 4. Including waist circumference or BMI-for-age $Z$-score as mediators, instead of FMI, confirmed the results (data not shown).

\section{Discussion}

\section{Summary}

The present study showed that shorter parental education was associated with higher insulin resistance and a more adverse lipid profile in Danish 8- to 11-year-olds. As expected, body fatness was an important explanatory factor in these associations. However, as a novel finding, whole-blood n-3 LCPUFA and dietary fibre intake were also seen to mediate part of the educational differences in some of the cardiometabolic risk markers. This indicates that educational differences in intakes of fish and dietary fibre affect cardiometabolic differences as early as in childhood.

\section{Interpretation and comparison with previous studies}

To our knowledge, no previous studies have investigated whether socio-economic differences in fish intake, wholeblood n-3 LCPUFA or dietary fibre intake serve as links between socio-economic position and cardiometabolic risk markers. n-3 LCPUFA supplementation consistently lowers plasma TAG in adults ${ }^{(42)}$ and has been shown to improve insulin resistance in obese children ${ }^{(43)}$. Also, previous studies have found an association between cheek-cell $n$-3 LCPUFA and the degree of social deprivation in children ${ }^{(44)}$ and erythrocyte $n-3$ LCPUFA and level of education in adults ${ }^{(45)}$. Altogether, this indirect evidence supports our finding that whole-blood $n-3$ LCPUFA has an explanatory role in the association between socioeconomic position and cardiometabolic risk markers. Oily fish is the main dietary source of $n-3$ LCPUFA in Danes, but fish-oil consumption may also contribute. In our study, an educational gradient was present in fish consumption, whereas this was not the case for fish-oil intake. Also, $76 \%$ of the children consumed fish, whereas only $15 \%$ consumed fish oil. Therefore, fish intake was most likely the main contributor to the educational differences in whole-blood $n$-3 LCPUFA.

Higher intake of dietary fibre has been associated with a reduced risk of overweight, obesity and impaired glucose metabolism in children ${ }^{(14)}$ and lower risk of type 2 diabetes in adults ${ }^{(46)}$. Low intake of dietary fibre may increase insulin resistance by contributing to overweight. However, to our surprise, we found that dietary fibre and wholeblood $n$-3 LCPUFA explained part of the difference in HOMA-IR between the children of parents with the shortest and longest educations, independent of body fatness. Similarly, whole-blood $n$-3 LCPUFA explained part of the educational differences in TAG independent of body fatness. This indicates that whole-blood $n$ - 3 LCPUFA and dietary fibre have an explanatory role in relation to cardiometabolic inequalities in children in addition to overweight. Still, our results showed that educational differences in overweight are important as a mediating factor. Moreover, body fatness was the only presumed mediator of the association between shorter parental education and higher total and LDL cholesterol. In support of this finding, increased BMI has been shown to increase childhood concentrations of total and LDL cholesterol ${ }^{(47)}$.

Differences in HOMA-IR and TAG were observed only between the reference group and the lower secondary and short higher education groups, respectively, the latter two being relatively small groups. Although the small size of the education groups increases the uncertainty of the estimates, the educational differences were comparable to those observed in previous studies ${ }^{(6,48)}$. In contrast, no educational differences were seen in blood pressure. A possible explanation could be a larger variation in this measure compared with the risk markers measured in blood.

\section{Generalizability and implications for public bealth}

The distribution of children in the education groups matched the distribution in Danish families with children fairly well, except that the lower secondary education group was somewhat under-represented, comprising 5\% in our study v. $10 \%$ among all Danish families with children ${ }^{(49)}$. Therefore, the estimates for this group may be more prone to bias than is the case for the other education groups. However, in the case of bias, the estimated differences between this group and the long higher education group would probably be underestimated as the children enrolled from the lower secondary education group were likely to be among the most advantaged children from that education group in the background population. The proportion of immigrant/descendants matched the $10 \%$ reported in the total Danish population ${ }^{(50)}$, the prevalence of overweight and obesity corresponded to that found in a recent cohort of Danish schoolchildren ${ }^{(51)}$ and the diet of the children was similar to that reported among children in the representative Danish National Dietary Survey ${ }^{(52)}$. This indicates that the study population was, to a large degree, representative of Danish 8- to 11-year-olds and that the results may be generalizable.

The immediate clinical implications of 0.36 point higher HOMA-IR and $0.12 \mathrm{mmol} / \mathrm{l}$ higher plasma TAG in children of parents with the shortest compared with the longest education are uncertain. However, blood lipids show tracking from childhood to adulthood ${ }^{(3)}$ and metabolic dysregulations during childhood may increase the risk of metabolic syndrome and diabetes in adulthood ${ }^{(53)}$. Thus, since atherosclerosis is a gradual, lifelong process, it is probable that a reduction in socio-economic differences in cardiometabolic risk markers in childhood will beneficially affect later inequalities in CVD risk. As indicated by our results, not only educational differences in overweight but also in consumption of fish and dietary fibre per se were 
Table 4 Differences $(\beta)$ in cardiometabolic risk markers in 8- to 11-year-old Danish children between the three groups with the shortest educations and the long higher education group (reference), and estimated mediated effects in absolute values and percentagest, 2011

\begin{tabular}{|c|c|c|c|c|c|c|c|c|c|}
\hline \multirow{5}{*}{ Cardiometabolic marker } & \multicolumn{9}{|c|}{ Highest level of education in the household } \\
\hline & \multicolumn{3}{|c|}{ Lower secondary ( $\leq 10$ years) } & \multicolumn{3}{|c|}{ Vocational (12 years) } & \multicolumn{3}{|c|}{ Short higher (14 years) } \\
\hline & $\beta$ & $95 \% \mathrm{Cl}$ & & $\beta$ & $95 \% \mathrm{Cl}$ & & $\beta$ & $95 \% \mathrm{Cl}$ & \\
\hline & \multicolumn{3}{|c|}{ Mediated effect } & \multicolumn{3}{|c|}{ Mediated effect } & \multicolumn{3}{|c|}{ Mediated effect } \\
\hline & Absolute value & $95 \% \mathrm{Cl}$ & $\%$ & Absolute value & $95 \% \mathrm{Cl}$ & $\%$ & Absolute value & $95 \% \mathrm{Cl}$ & $\%$ \\
\hline $\begin{array}{l}\text { TC }(\mathrm{mmol} / \mathrm{l}) \\
\text { FMl } \\
\text { LDL-C }(\mathrm{mmol} / \mathrm{l}) \\
\text { FMl }\end{array}$ & & & & $\begin{array}{l}0 \cdot 14^{\star} \\
0 \cdot 03^{\star} \\
0 \cdot 14^{\star} \\
0.04^{\star \star}\end{array}$ & $\begin{array}{l}0.02,0.27 \\
0.00,0.06 \\
0.03,0.25 \\
0.01,0.07\end{array}$ & $\begin{array}{l}- \\
21 \\
- \\
29\end{array}$ & & & \\
\hline TAG $(\mathrm{mmol} / \mathrm{l})$ & $0.12^{\star \star}$ & $0.04,0.21$ & - & & & & $0.08^{*}$ & $0.01,0.14$ & - \\
\hline FMI & $0.03^{\star *}$ & $0.01,0.05$ & 25 & & & & $0.02^{* *}$ & $0.01,0.03$ & 25 \\
\hline n-3 LCPUFA & $0.02^{\star \star}$ & $0.00,0.04$ & 17 & & & & $0.01^{\star *}$ & $0.00,0.02$ & 13 \\
\hline FMI and $n-3$ LCPUFA & $0.05^{\star \star \star}$ & $0.03,0.08$ & 42 & & & & $0.03^{\star * *}$ & $0.01,0.04$ & 38 \\
\hline HOMA-IR $\ddagger$ & $0.36^{\star \star}$ & $0.10,0.62$ & - & & & & & & \\
\hline FMI & $0.23^{\star \star \star}$ & $0.11,0.35$ & 64 & & & & & & \\
\hline Dietary fibre§ & $0 \cdot 12^{*}$ & $0.01,0.22$ & 33 & & & & & & \\
\hline$n$-3 LCPUFA & $0.07^{*}$ & $0.02,0.13$ & 19 & & & & & & \\
\hline Dietary fibre, $n$-3 LCPUFA§ & $0 \cdot 17^{\star \star}$ & $0.05,0.29$ & 47 & & & & & & \\
\hline FMI, dietary fibre, $n$-3 LCPUFA§ & $0.32^{* * *}$ & $0.17,0.47$ & 89 & & & & & & \\
\hline
\end{tabular}

TC, total cholesterol; FMI, fat mass index; LDL-C, LDL cholesterol; $n-3$ LCPUFA, $n-3$ long-chain PUFA (w/w\% of the total fatty acids measured in whole blood); HOMA-IR, homeostasis model assessment of insulin resistance.

$P<0.01,{ }^{* \star *} P<0.001$

†All models were adjusted for school, school class, sex, age, height, puberty, a sex $\times$ puberty interaction and fasting status. TAG and HOMA-IR were log-transformed in the analyses, but the results are presented on the original scales.

Rere available for 675 children only: lower secondary ( $n$ 31), vocational ( $n$ 236), short higher $(n 68)$, medium higher $(n$ 198) and long higher $(n 142)$

$\S$ Additionally adjusted for under-, acceptable and over-reporting of energy intake. 
possible explanations for differences in cardiometabolic risk markers and may therefore be important targets in public health strategies to prevent inequalities in children's cardiometabolic health.

\section{Strengths and limitations}

A major strength of the present study is the use of body fatness by dual-energy X-ray absorptiometry scan, a biomarker of fish intake and objectively measured physical activity. Whole-blood $n$-3 LCPUFA, instead of reported fish intake, was a relevant mediator to include in order to overcome potential misreporting and recall bias. Twentyfour per cent of the children reported not having eaten fish during the $7 \mathrm{~d}$ recording period, but they may have eaten fish during previous weeks. The response of whole-blood fatty acids to $n-3$ LCPUFA supplementation has been shown to be intermediate between that of plasma and erythrocyte fatty acids, which reflect intakes over the last days to weeks and over the last months, respectively ${ }^{(54)}$. Whole-blood $n$-3 LCPUFA correlated fairly well with fish intakes and, compared with erythrocytes, whole blood required less processing in the laboratory truck. Physical activity was measured by accelerometers that have a much higher validity than self-reported physical activity ${ }^{(55)}$. As the categorization of minutes spent in MVPA has not been validated for triaxial data, we used the vertical axis only with a position capturing movements by the centre of mass. The advantage of this position is a relatively close relationship with energy expenditure, whereas the disadvantage is a limited ability to capture bicycling, which often belongs to the MVPA category. Only $82 \%$ of the children reported time spent bicycling, therefore we could not include this parameter in the analyses.

We used a relatively detailed grouping of education, as we wanted to explore whether the educational differences were general trends in the population or related to particular, vulnerable groups. As a limitation, this approach precluded detailed investigation of the interplay between ethnicity, sex and parental education. Under-reporting of energy intake was higher in the lower secondary education group but was adjusted for in the analyses and has therefore probably not affected the results substantially. We also acknowledge that the many statistical tests performed give an increased risk of false positive findings; however, due to the exploratory nature of the study, adjustment for multiplicity was not applied. Finally, the use of cross-sectional data does not allow conclusions on causality. However, the risk of reverse causality is low, as it seems unlikely that children's cardiometabolic profiles influence their dietary habits or their parents' education levels, and the potential mediators were chosen based on previous evidence showing that they affect the cardiometabolic risk markers. Still, residual confounding, by, for example, other dietary components or psycho-social influences, cannot be precluded.

\section{Conclusions}

Among Danish 8- to 11-year-olds, children of parents with shorter compared with long educations had higher insulin resistance and less favourable lipid profiles. Body fatness, whole-blood n-3 LCPUFA and dietary fibre intake, but not MVPA or sedentary time, were independent mediators of these differences. The mediators explained a greater proportion of the educational differences when combined than when assessed individually. Therefore, not only prevention of overweight but also increasing the intakes of fish and dietary fibre among children of parents with shorter educations could be potential targets for public health interventions to reduce socio-economic inequalities in cardiometabolic risk from childhood. However, as these cross-sectional results do not justify conclusions on causality, confirmation in longitudinal studies is warranted.

\section{Acknowledgements}

Acknowledgements: The authors are grateful to the participating children, their families, the school administrators, teachers and other staff, and thank the entire study team for their contribution to this work. Financial support: The OPUS School Meal Study was supported by the Nordea Foundation (grant number 02-2010-0389). The Foundation did not have any role in the design, data collection, analysis, interpretation of results or the decision to publish. Conflict of interest: None. Authorship: H.H. designed and performed the statistical analysis and wrote the manuscript. M.V.G. designed the analyses and helped interpret the data. C.R. supervised the statistical analysis and helped write the statistics section and interpret the data. A.B.-J. and R.A. collected and processed the dietary data. S.-M.D. collected the data and processed data related to body fatness. M.F.H. collected and processed the physical activity data. A.S. designed the research and supervised the collection of the physical activity data. A.A. and K.F.M. designed the research and supervised the data collection. C.T.D. designed the research and analysis, interpreted the data and helped write the manuscript. All authors critically reviewed the manuscript and approved the final version. Ethics of human subject participation: The study was conducted according to the principles of the Declaration of Helsinki and approved by the Committees on Biomedical Research Ethics for the Capital Region of Denmark (H-1-2010-124), and written informed consent was obtained from all custody holders of the children.

\section{References}

1. Mackenbach JP, Stirbu I, Roskam AR et al. (2008) Socioeconomic inequalities in health in 22 European countries. N Engl J Med 358, 2468-2481. 
2. Berenson G, Srinivasan S, Bao W et al. (1998) Association between multiple cardiovascular risk factors and atherosclerosis in children and young adults. $N$ Engl J Med 338, 1650-1656.

3. Juhola J, Magnussen CG, Viikari JSA et al. (2011) Tracking of serum lipid levels, blood pressure, and body mass index from childhood to adulthood: the Cardiovascular Risk in Young Finns Study. J Pediatr 159, 584-590.

4. Pollitt RA, Rose KM \& Kaufman JS (2005) Evaluating the evidence for models of life course socioeconomic factors and cardiovascular outcomes: a systematic review. BMC Public Health 5, 7.

5. Howe L, Galobardes B, Sattar N et al. (2010) Are there socioeconomic inequalities in cardiovascular risk factors in childhood, and are they mediated by adiposity? Findings from a prospective cohort study. Int J Obes (Lond) $\mathbf{3 4}$, 1149-1159.

6. Lawlor DA, Harro M, Wedderkopp $\mathrm{N}$ et al. (2005) Association of socioeconomic position with insulin resistance among children from Denmark, Estonia, and Portugal: cross sectional study. BMJ 331, 183.

7. van den Berg G, van Eijsden M, Vrijkotte TG et al. (2012) Socioeconomic inequalities in lipid and glucose metabolism in early childhood in a population-based cohort: the ABCDStudy. BMC Public Health 12, 591.

8. Goodman E, McEwen BS, Huang B et al. (2005) Social inequalities in biomarkers of cardiovascular risk in adolescence. Psychosom Med 67, 9-15.

9. Weiss R, Dziura J, Burgert TS et al. (2004) Obesity and the metabolic syndrome in children and adolescents. $N$ Engl J Med 350, 2362-2374.

10. Shrewsbury V \& Wardle J (2008) Socioeconomic status and adiposity in childhood: a systematic review of crosssectional studies 1990-2005. Obesity (Silver Spring) 16, 275-284.

11. Goodman E, Must A, Daniels SR et al. (2010) Hostility and adiposity mediate disparities in insulin resistance among adolescents and young adults. J Pediatr 157, 572-577.

12. Wells J, Cole T \& ALSPAC study team (2002) Adjustment of fat-free mass and fat mass for height in children aged 8 y. Int J Obes Relat Metab Disord 26, 947-952.

13. Ambrosini GL, Huang RC, Mori TA et al. (2010) Dietary patterns and markers for the metabolic syndrome in Australian adolescents. Nutr Metab Cardiovasc Dis 20, 274-283.

14. Brauchla M, Juan W, Story J et al. (2012) Sources of dietary fiber and the association of fiber intake with childhood obesity risk (in 2-18 year olds) and diabetes risk of adolescents 12-18 year olds: NHANES 2003-2006. J Nutr Metab 2012, 736258.

15. Hartley L, Igbinedion E, Holmes J et al. (2013) Increased consumption of fruit and vegetables for the primary prevention of cardiovascular diseases. Cochrane Database Syst Rev 6, CD009874.

16. Whelton SP, Hyre AD, Pedersen B et al. (2005) Effect of dietary fiber intake on blood pressure: a meta-analysis of randomized, controlled clinical trials. I Hypertens $\mathbf{2 3}$, 475-481.

17. He K, Song Y, Daviglus ML et al. (2004) Accumulated evidence on fish consumption and coronary heart disease mortality: a meta-analysis of cohort studies. Circulation 109, 2705-2711.

18. Kotwal S, Jun M, Sullivan D et al. (2012) Omega 3 fatty acids and cardiovascular outcomes systematic review and meta-analysis. Circ Cardiovasc Qual Outcomes 5 , 808-818.

19. Brug J, van Stralen MM, te Velde SJ et al. (2012) Differences in weight status and energy-balance related behaviors among schoolchildren across Europe: the ENERGY-Project. PLoS One 7, e34742.
20. Rasmussen M, Krølner R, Klepp K et al. (2006) Determinants of fruit and vegetable consumption among children and adolescents: a review of the literature. Part I: quantitative studies. Int J Behav Nutr Phys Act 3, 22.

21. Ekelund U, Luan J, Sherar LB et al. (2012) Moderate to vigorous physical activity and sedentary time and cardiometabolic risk factors in children and adolescents. JAMA 307, 704-712.

22. Ford ES \& Caspersen CJ (2012) Sedentary behaviour and cardiovascular disease: a review of prospective studies. Int J Epidemiol 41, 1338-1353.

23. Damsgaard CT, Dalskov S, Petersen RA et al. (2012) Design of the OPUS School Meal Study: a randomised controlled trial assessing the impact of serving school meals based on the New Nordic Diet. Scand J Public Health 40, 693-703.

24. Groth MV, Fagt S, Stockmarr A et al. (2009) Dimensions of socioeconomic position related to body mass index and obesity among Danish women and men. Scand J Public Health 37, 418-426.

25. Groth M, Christensen L, Knudsen V et al. (2013) Social Differences in Children's Dietary Habits, Physical Activity and Overweight, and Adult's Dietary Habits. Copenhagen: Technical University of Denmark, National Food Institute, Division of Nutrition (in Danish with an English summary).

26. Winkleby MA, Jatulis DE, Frank E et al. (1992) Socioeconomic status and health: how education, income, and occupation contribute to risk factors for cardiovascular disease. Am J Public Health 82, 816-820.

27. Statistics Denmark (2008) Forspalte 1 - uddannelsesnomenklaturer (Standard classifications of education). http://www.dst.dk/da/Statistik/dokumentation/Nomenklaturer/ Forspalter.aspx (accessed July 2013).

28. Morris NM \& Udry JR (1980) Validation of a selfadministered instrument to assess stage of adolescent development. J Youth Adolesc 9, 271-280.

29. Biltoft-Jensen A, Trolle E, Christensen T et al. (2014) WebDASC: a web-based dietary assessment software for 8-11year-old Danish children. J Hum Nutr Diet 27, Suppl. 1, 43-53.

30. Henry CJK (2005) Basal metabolic rate studies in humans: measurement and development of new equations. Public Health Nutr 8, 1133-1152.

31. Black AE (2000) The sensitivity and specificity of the Goldberg cut-off for EI:BMR for identifying diet reports of poor validity. Eur J Clin Nutr 54, 395-404.

32. Hjorth MF, Chaput J, Michaelsen K et al. (2013) Seasonal variation in objectively measured physical activity, sedentary time, cardio-respiratory fitness and sleep duration among 8-11 year-old Danish children: a repeatedmeasures study. BMC Public Health 13, 808.

33. Trost SG, Loprinzi PD, Moore R et al. (2011) Comparison of accelerometer cut points for predicting activity intensity in youth. Med Sci Sports Exerc 43, 1360-1368.

34. de Onis M., Onyango AW, Borghi E et al. (2007) Development of a WHO growth reference for school-aged children and adolescents. Bull World Health Organ 85, 660-667.

35. Cole TJ, Flegal KM, Nicholls D et al. (2007) Body mass index cut offs to define thinness in children and adolescents: international survey. BMJ 335, 194.

36. Cole TJ, Bellizzi MC, Flegal KM et al. (2000) Establishing a standard definition for child overweight and obesity worldwide: international survey. BMJ 320, 1240-1243.

37. Damsgaard CT, Dalskov S, Laursen RP et al. (2014) Provision of healthy school meals does not affect the metabolic syndrome score in 8-11-year-old children, but reduces cardiometabolic risk markers despite increasing waist circumference. Br J Nutr 112, 1826-1836.

38. Damsgaard CT, Eidner MB, Stark KD et al. (2014) Eicosapentaenoic acid and docosahexaenoic acid in whole 
blood are differentially and sex-specifically associated with cardiometabolic risk markers in 8-11-year-old Danish children. PLoS One 9, e109368.

39. Baron RM \& Kenny DA (1986) The moderator-mediator variable distinction in social psychological research: conceptual, strategic, and statistical considerations. J Pers Soc Psychol 51, 1173-1182.

40. Laursen RP, Dalskov S, Damsgaard CT et al. (2014) Back-transformation of treatment differences - an approximate method. Eur J Clin Nutr 68, 277-280.

41. R Core Team (2013) A Language and Environment for Statistical Computing. Vienna: R Foundation for Statistical Computing; available at http://www.R-project.org/

42. Mozaffarian D \& Wu JH (2011) Omega-3 fatty acids and cardiovascular disease: effects on risk factors, molecular pathways, and clinical events. J Am Coll Cardiol 58, 2047-2067.

43. López-Alarcón M, Martínez-Coronado A, Velarde-Castro O et al. (2011) Supplementation of $\mathrm{n} 3$ long-chain polyunsaturated fatty acid synergistically decreases insulin resistance with weight loss of obese prepubertal and pubertal children. Arch Med Res 42, 502-508.

44. Kirby A, Woodward A, Jackson S et al. (2010) Children's learning and behaviour and the association with cheek cell polyunsaturated fatty acid levels. Res Dev Disabil 31, 731-742.

45. Cohen BE, Garg SK, Ali S et al. (2008) Red blood cell docosahexaenoic acid and eicosapentaenoic acid concentrations are positively associated with socioeconomic status in patients with established coronary artery disease: data from the Heart and Soul Study. J Nutr 138, 1135-1140.

46. Schulze MB, Schulz M, Heidemann C et al. (2007) Fiber and magnesium intake and incidence of type 2 diabetes: a prospective study and meta-analysis. Arch Intern Med 167, 956-965.
47. Dai S, Fulton JE, Harrist RB et al. (2009) Blood lipids in children: age-related patterns and association with body-fat indices: Project HeartBeat! Am J Prev Med 37, 1 Suppl., S56-S64.

48. Thomas C, Nightingale CM, Donin AS et al. (2012) Socio-economic position and type 2 diabetes risk factors: patterns in UK children of South Asian, Black AfricanCaribbean and White European origin. PLoS One 7, e32619.

49. Statistics Denmark (2002) StatBank Denmark: Income, consumption and prices. INDKF7 2011. http://www. statistikbanken.dk/INDKF7 (accessed April 2013).

50. Statistics Denmark (2012) Indvandrere i Danmark 2012 (Immigrants in Denmark 2012). http://www.dst.dk/ pukora/epub/upload/16601/indv.pdf (accessed September 2013).

51. Pearson S, Hansen B, Sørensen TI et al. (2010) Overweight and obesity trends in Copenhagen schoolchildren from 2002 to 2007. Acta Paediatr 99, 1675-1678.

52. Pedersen AN, Fagt S, Groth MV et al. (2010) Dietary Habits in Denmark 2003-2008: Main Results. Copenhagen: National Food Institute, Division of Nutrition (in Danish with an English summary).

53. Morrison JA, Friedman LA, Wang P et al. (2008) Metabolic syndrome in childhood predicts adult metabolic syndrome and type 2 diabetes mellitus 25 to 30 years later. J Pediatr 152, 201-206.

54. Metherel AH, Armstrong JM, Patterson AC et al. (2009) Assessment of blood measures of $n-3$ polyunsaturated fatty acids with acute fish oil supplementation and washout in men and women. Prostaglandins Leukot Essent Fatty Acids 81, 23-29.

55. Westerterp KR (2009) Assessment of physical activity: a critical appraisal. Eur J Appl Physiol 105, 823-828. 